BULL. AUSTRAL. MATH. SOC.

\title{
Uniqueness and representation of a function in terms of its translated averages
}

\section{Chin-Hung Ching}

We give the representation formula of a periodic function with period one in terms of its translated means on the unit interval. We also give an application of this formula to a boundary value problem.

Let $f$ be a continuous periodic function on the real line with period one. We consider its a means

$$
s_{\alpha, n}(f)=\frac{1}{n} \sum_{k=1}^{n} f\left(\alpha+\frac{k}{n}\right)
$$

$n=1,2, \ldots$, and

$$
s_{\alpha, \infty}(f)=\lim _{n \rightarrow \infty} s_{\alpha, n}(f)
$$

We denote

$$
R_{\alpha, n}(f)=S_{\alpha, n}(f)-S_{\alpha, \infty}(f)
$$

As.in [5], a periodic function $f$ with period one is defined to be in $B^{\mathcal{E}}$ if the Fourier coefficients of $f$ satisfy the following condition:

$$
a_{n}(f)=\int_{0}^{1} f(t) e^{-i 2 \pi n t} d t=o\left(\frac{1}{n}\right) .
$$

It is easy to prove that $a_{n}(f)$ and $R_{\alpha, n}(f)$ have the same rate of Received 20 March 1973. 
convergence to zero, (cf. [4]). Let $\mu(n)$ be the Möbius function of $n$. We announce the following results.

THEOREM 1. Let $\alpha$ and $\beta$ be any two real numbers in $[0,1]$ such that $\alpha-\beta$ is irrational and $f$ be a periodic continuous function with period one such that $s_{\alpha, n}(f)=S_{\beta, n}(f)=0$ for all $n>0$. Then $f$ is the zero function if $f$ satisfies one of the following conditions:

(a) $f \in B^{s}, s>1$;

(b) $f$ has bounded variation in $[0,1]$;

(c) $\sum_{|k|=N}^{\infty}\left|a_{n}\right|=O\left(\frac{1}{N}\right)$;

(d) the Fourier series of $f$ is an absolutely convergent gap series.

In case $f$ can be regarded as the boundary value of an analytic function, an analogous uniqueness result has been announced in [2] and proved in [3] and [6]. Also if $\alpha-\beta$ is rational, it is easy to find a function $g$ such that $S_{\alpha, n}(g)=S_{\beta, n}(g)=0$ for all integers $n$.

THEOREM 2. Let $\alpha$ and $\beta$ be any two real numbers in $[0,1]$.

(i) Suppose $\alpha-\beta$ is irrational and $f$ is in $B^{s}$ with $s>1$; then

$$
a_{m}(f)=\sum_{k=1}^{\infty} \frac{\mu(k)\left[e^{-i 2 \pi m \beta_{R}} \alpha_{\alpha,|m k|}(f)-e^{-i 2 \pi m \alpha_{R}} R_{B,|m k|}(f)\right]}{2 i \sin 2 \pi m(\alpha-\beta)}
$$

for all $m= \pm 1, \pm 2, \ldots$.

(ii) Let $\alpha-\beta$ be an algebraic number of degree $q \geq 2$ and $f$ be in $B^{s}$ with $s>\sqrt{2 q}$. Then

(1) $f(t)=R_{\alpha, \infty}(f)+\sum_{n=1}^{\infty}\left[R_{\alpha, n}(f) P_{n}(\alpha, \beta, t)+R_{\beta, n}(f) P_{n}(\beta, \alpha, t)\right]$, where the series converges uniformly with the rate $N^{x-s}, \sqrt{2 q}<x<s$, and 


$$
P_{n}(\alpha, \beta, t)=\sum_{j\lceil n} \mu\left(\frac{n}{j}\right) \frac{\sin 2 \pi j(t-\beta)}{\sin 2 \pi j(\alpha-\beta)} .
$$

Results analogous to Theorem 2 (ii) in the case that $f$ is the boundary value of an analytic function were announced in [1] and established in [5].

THEOREM 3. Let $\alpha$ and $\beta$ be any two real numbers in $[0,1]$ such that $\alpha-\beta$ is algebraic and let $f$ be in $B^{s}$ for any $s>0$, then

$$
\begin{array}{r}
R_{\alpha, m}\left(f^{\prime}\right)=S_{\alpha, m}\left(f^{\prime}\right)=2 \pi m \sum_{k=1}^{\infty} \sum_{\zeta k} Z \mu\left(\frac{k}{2}\right)\left[R_{\alpha, k m}(f) \cot 2 \pi Z m(x-\beta)\right. \\
\left.-R_{\beta, k m}(f) \csc 2 \pi 2 m(\alpha-\beta)\right],
\end{array}
$$

$R_{\alpha, m}\left(f^{\prime \prime}\right)=-4 \pi^{2} m^{2} \sum_{k=1}^{\infty} R_{\alpha, k m}(f)\left[\sum_{\tau\lceil k} \mu\left(\frac{k}{\tau}\right) z^{2}\right]$.

As $f$ is uniquely determined by the averages $\left\{R_{\alpha, n}(f)\right\}$ and $\left\{R_{B, n}(f)\right\}$, consequently $R_{\alpha, m}\left(f^{\prime}\right)$ and $R_{\beta, m}\left(f^{\prime}\right)$ depend also on these averages. However it is not clear from the definition that $R_{\alpha, m}\left(f^{\prime}\right)$ and $R_{\beta, m}\left(f^{\prime}\right)$ depend only on those $R_{\alpha, k}(f)$ and $R_{B, k}(f)$ such that $k$ is a multiple of $m$.

We also remark that $P_{n}(\alpha, B ; t)$ has the property that $S_{\beta, m}\left[P_{n}(\alpha, \beta ; t)\right]=0$ and $S_{\alpha, m}\left[P_{n}(\alpha, \beta ; t)\right]=\delta_{m, n}$ for all positive integers $m$ and $n$. The assumption on $\alpha-\beta$ is not satisfactory, yet we guess that the series (1) will diverge if $\alpha-\beta$ is a Liouville number, (cf. [7]). Now we give an application of the above theorems to a boundary value problem.

Let $\alpha$ and $\beta$ be any two real numbers such that $\alpha-\beta$ is an algebraic number of degree $q \geq 2$. Let $u(t) \in B^{s}, s>\sqrt{2 q}-1$, be the solution of the following boundary value problem:

$$
\begin{aligned}
a u^{\prime \prime}+b u^{\prime}+c u & =f ; f \in B^{p}, p>1 ; \\
u(0) & =u(1), \\
u^{\prime}(0) & =u^{\prime}(1),
\end{aligned}
$$


where $a, b, c$ are constants satisfying

$$
c+2 \pi i n b-4 \pi^{2} n^{2} a \neq 0
$$

for all $n=0, \pm 1, \ldots$. Then

(2) $R_{\alpha, \infty}(u)=R_{\beta, \infty}(u)=\frac{1}{c} R_{\alpha, \infty}(f)$,

$$
\begin{aligned}
& R_{\alpha, n}(u)=\sum_{k=1}^{\infty} R_{\alpha, k n}(f)\left|\sum_{\nu \mid k} \mu\left(\frac{k}{\tau}\right) \frac{\left(c-4 \pi^{2} \eta^{2} n^{2} a\right)-2 \pi \eta n b \cot 2 \pi \eta n(\alpha-\beta)}{c^{2}-8 \pi^{2} \eta^{2} n^{2} a+4 \pi^{2} \eta^{2} n^{2} b^{2}+16 \pi^{4} \eta^{4} n^{4} a^{2}}\right| \\
& +\sum_{k=1}^{\infty} R_{\beta, k n}(f)\left|\sum_{\eta\} k} \mu\left(\frac{k}{l}\right) \frac{2 \pi l n b c s c 2 \pi z n(\alpha-\beta)}{c^{2}-8 \pi^{2} z^{2} n^{2} a+4 \pi^{2} z^{2} n^{2} b^{2}+16 \pi^{4} z^{4} n^{4} a^{2}}\right|
\end{aligned}
$$

It follows from the above formula that if for fixed $j,\left\{R_{\alpha, j n}(f)\right\}$ and $\left\{R_{B, j n}(f)\right\}$ are known for all $n=1,2, \ldots$, then $\left\{R_{\alpha, j n}(u)\right\}$ and $\left\{R_{B, j n}(u)\right\}$ can be computed from (2). However we can see from Theorem 2 (ii) that if $j \neq 1$, then $\left\{R_{\alpha, j n}(f)\right\}$ and $\left\{R_{\beta, j n}(f)\right\}$ do not determine $f$ uniquely. In other words we can find some averages of the output from a portion of the averages of the input. The details of the proof of the theorems and other related results will appear elsewhere.

\section{References}

[1] Chin-Hung Ching and Charles K. Chui, "Representation of a function in terms of its mean boundary values", Bulz. Austral. Math. Soc. 7 (1972), 425-427.

[2] Chin-Hung Ching and Charles K. Chui, "Uniqueness and nonuniqueness in mean boundary value problems", Bull. Austral. Math. Soc. 8 (1973), 23-26.

[3] Chin-Hung Ching and Charles K. Chui, "Analytic functions characterized by their means on an arc", Trans. Amer. Math. Soc. (to appear).

[4] Chin-Hung Ching and Charles K. Chui, "Axymptotic similarities of Fourier and Riemann coefficients", J. Approximation Theory (to appear). 
[5] Chin-Hung Ching and Charles K. Chui, "Mean boundary value problems and Riemann series", J. Approximation Theory (to appear).

[6] Chin-Hung Ching and Charles K. Chui, "Uniqueness theorems determined by function values at the roots of unity", $J$. Approximation Theory (to appear).

[7] I van Niven, Irrational numbers (Carus Mathematical Monographs, No. 11. Mathematical Association of America; John Wiley \& Sons, New York, 1956).

Department of Mathematics, Texas A\&M University, College Station, Texas,

USA.

Present Address:

Department of Mathematics, University of Melbourne,

Parkville,

victoria. 